\title{
A model of heritage content to support the design and analysis of video games for history education
}

\author{
Laurence Hanes ${ }^{1}$ - Robert Stone ${ }^{1}$
}

Received: 13 April 2018/Revised: 9 July 2018/Accepted: 11 September 2018/

Published online: 18 September 2018

(C) The Author(s) 2018

\begin{abstract}
In recent years, increasing academic attention has been focused on the educational potential of video games. Serious games for heritage applications have received much interest, and many examples are well documented in the literature. Many frameworks and methodologies have been proposed for how to design serious games, yet there is a lack of guidance for how to embed heritage information in the mechanics and content of these games. There are also many examples of commercial (non-serious) games that contain heritage content, and there is interest in how these games can be used in educational contexts. However, these games are designed primarily for entertainment purposes, without pedagogical foundations and with limited historical accuracy. There is currently a lack of a rigorous, scientific approach to support the critical analysis of the content of these games and to inform their use within learning settings. In this paper, we propose that the above issues are related by a lack of definitions of heritage content in the video game medium. We describe a model that defines how historical information can be presented in a video game at a content level and demonstrate how it can be applied to the analysis of the content in a commercial historical game. Finally, we propose a novel methodology based on activity theory to guide the design of serious games, based on pre-defined heritage instructional content. The findings are relevant both to educators who wish to use heritage video games, and to designers of serious games for heritage.
\end{abstract}

Keywords Virtual heritage - Serious games · Game design · Game analysis

Laurence Hanes

LXH519@bham.ac.uk

1 School of Engineering, University of Birmingham, Birmingham B15 2TT, UK 


\section{Introduction}

Serious games (SGs) have been defined as "digital games with educational objectives" (Catalano et al. 2014) and combine the engaging and motivational characteristics of video games with educational outcomes based upon sound pedagogical principles (Dondlinger 2007). Over recent years, increasing focus has been applied to investigating whether these games can indeed achieve their instructional objectives in an engaging and entertaining manner (Girard et al. 2013; Connolly et al. 2012; Boyle et al. 2016; Kim et al. 2017). Serious games have been applied to a wide range of subjects, such as science (Cheng et al. 2015), healthcare (Beale et al. 2007), social enterprise (Damani et al. 2015) and history and heritage (Paliokas and Sylaiou 2016) which is the subject of interest in this paper.

There are now many examples of successfully implemented and tested serious games for heritage (SGsH; e.g. Bellotti et al. 2012; Chen et al. 2013; Christopoulos et al. 2011; Dagnino et al. 2015; Doulamis et al. 2012; Birchall and Henson 2011; Jacobson et al. 2009; Kelly and Bowan 2014; Kidd 2015; Perez-Valle et al. 2014; Shih et al. 2015); a comprehensive literature survey was presented by Paliokas and Sylaiou (2016). However, there is nevertheless a lack of frameworks and guidelines for how heritage content can be presented through serious games and how to design and develop SGsH.

In addition, there is also a strong trend within the commercial video game industry to use historical themes, settings, characters and events. Due to the mass appeal and prevalence of these types of games, there is an increasing interest in how these games can be used within the context of history education (Squire 2005; Watson et al. 2011; Pagnotti and Russell 2012; McCall 2016). These games however are prioritised to be entertaining, often with a tenuous relationship with historical accuracy (Copplestone 2017) and without defined learning objectives. In his comprehensive overview of the field, McCall (2016) stresses that educators wishing to use historical games need to be aware of the ways in which the games are or are not accurate, and he concludes that enumerating the heritage content within these games would be a valuable activity. However, he does not offer specific directions as to how to go about this.

We propose that these two issues are related at a fundamental level, and so share a common solution. The commonality of these issues is a lack of definitions of what heritage content can be manifested within the video game medium and how it can be presented. Such a definition should also highlight the ways in which heritage content in SGs differs from that presented in video games for entertainment. Hanes and Stone (2017) presented a model to give such a definition and in the present paper we will describe this model and show how it can be applied to critically evaluate the heritage content in an entertainment game for use in educational contexts. We will then propose a novel approach based on activity theory to apply the model to the design of SGsH. 


\section{Literature review}

\section{Definitions of heritage video games}

\section{Definitions of SGsH}

Anderson et al. (2010) presented a review of serious games for heritage and grouped them into three categories-prototypes and demonstrators, which aim to accurately reconstruct historical events, actors and sites, virtual museums, which aim to use gaming technology to recreate a traditional museum experience in a more engaging way, and commercial historical games, which are designed primarily for entertainment but which depict real historical events and so can be used in an educational context.

Mortara et al. (2014) presented a similar review of serious games for heritage but chose a different taxonomy to categorise the games they reviewed. They defined cultural awareness games, which present customs, cultures and intangible heritage, defined as "practices, representations, expressions, as well as the knowledge and skills, that communities, groups and, in some cases, individuals recognize as part of their cultural heritage" (UNESCO 2003). They also defined historical reconstruction games, which aim to accurately reconstruct historical sites and events, and heritage awareness games, which are further split into two categories. Artistic/ archaeological heritage games present tangible heritage and cultural artefacts, whereas architectural/natural heritage games present large-scale heritage such as buildings, architecture and landscapes.

Antoniou et al. (2013) defined a model based on several game-based, playerbased and organisational characteristics of serious games for heritage, such as the cognitive skills the game enhances, whether the player is alone or in a group, and the goals that the organisation wishes to achieve through the use of the game.

Schaller (2014) proposed two approaches to the design of serious games for heritage. Extrinsic design involves inserting the heritage informational content into proven and well-founded game mechanics and genres, whereas intrinsic design involves designing original mechanics from the informational content. He advises that the extrinsic approach entails less-risk but will produce less novel games, and that the intrinsic approach is high-risk but creates the possibility to produce novel and highly engaging games.

\section{Definitions of commercial historical video games}

In the context of applying commercial historical video games to education, McCall (2016) defined a spectrum of heritage games, where at one end the player assumes the role of an individual historical actor (for example in role-playing or action games), and at the other end the player assumes the role of a more powerful agent with no historical analogue (for example in strategy games where the player has access to information no single historical actor would have had). 
Similarly, Uricchio (2005) defined a spectrum of commercial historical games from games which represent a historically specific simulation (with its associated events, sites and actors) to games that present heritage as a nonspecific simulation based instead on historical processes (for example strategy games where the player's actions alter the course of history).

Finally, Chapman (2016) defined yet another spectrum between two styles of historical simulation. At one end, the realist simulation style attempts to reconstruct the past "as it was" through the perspective of a small number of historical characters, focusing on audio-visual presentation and borrowing tropes from media such as film. At the other end, the conceptual simulation style represents heritage content as procedural systems through the game rules and mechanics, often using simpler, abstracted visual presentations.

All of the presented definitions of SGsH and commercial historical video games share a limitation, whereby the game is treated as a single atomic unit and no definitions are given for how the heritage information is manifested through the mechanics and content of the game.

\section{Frameworks for serious games}

Other authors have compiled literature surveys (Lepe-Salazar 2015) or have presented comparisons of serious game conceptual frameworks (dos Santos and Fraternali 2015). The following review is not intended to be exhaustive but covers what we believe are the most relevant serious game frameworks and references within the field.

\section{Initial frameworks based on pedagogical foundations}

Earlier work on serious games saw the emergence of frameworks that have arguably helped to define the field of serious games and how they should be designed and evaluated, based on thorough pedagogical foundations.

Gunter et al. (2006) presented the RETAIN framework and argued that a successful serious game should intrinsically couple together gaming and learning activities, and that the learning must be guided by established pedagogical principles. The framework is formed from a set of elements the designers of SGs should consider, to help guide the design and evaluation processes, based on Bloom's Taxonomy (1956) and Krathwohl et al. (1973), Keller's ARCS (Attention, Relevance, Confidence, Satisfaction) model (1983) and Gagne's 9 Events of Instruction (1985). The authors also showed how the framework could be applied to the analysis of existing SGs (Gunter et al. 2008).

The 4-Dimensional Framework (4DF) was presented by De Freitas and Oliver (2006) and attempts to model learning in video games using four interacting dimensions - the context in which the learning takes place, the attributes of the learners, the internal representations used by the video game, and the pedagogic approaches and processes of learning taken.

Finally, Amory (2007) proposed the Game Object Model II (GOM II), a framework for serious games based on an object-oriented paradigm, centred around 
six categories-game definition, authentic learning, narrative, gender, social collaboration and challenges-puzzles-quests.

\section{High-level conceptual frameworks}

More recently, researchers have proposed frameworks to describe the composition of SGs and how players learn with them from a theoretical perspective. Yusoff et al. (2009) proposed such a conceptual framework to describe the composition of SGs, presented as a class diagram. The authors attempted to validate their model experimentally using the Technology Acceptance Model (Yusoff et al. 2010).

Landers (2014) identified an overlap (and hence duplicated effort) in the fields of serious games and gamification of learning, and presented a unified "theory of gamified learning" to address this. In this theory, different game elements affect the player's behaviours and attitudes, which either directly moderates learning, improving its quality, or indirectly mediates it, by causing other behaviours which result in improved learning.

The Three-Dimensional Model was presented by Degens et al. (2015) and is based upon three concepts; the game, the user and the learning, but focuses on the interactions between each of those concepts. They show how game-user interactions, game-learning interactions and user-learning interactions should be carefully considered to assist in both the evaluation and design of successful educational games.

\section{Implementation-focused frameworks}

Conversely, other authors have presented frameworks that focus on giving practical guidelines for designing and evaluating SGs. Marne et al. (2012) applied their library of design patterns to their previously published conceptual framework, the Six Facets Model (Ibáñez et al. 2011), which is based upon six fundamental aspects of serious games-pedagogical objectives, domain simulation, interactions with the simulation, problems and progression, decorum and conditions of use. They give guidance on how to apply the design patterns to an SG such that each of the six facets are adequately reinforced.

Lepe-Salazar (2015) presented GAGE (Goal, Audience, Game, Environment), a model based on seven categories - the stakeholders, game goal, audience, game, environment, enhancing the experience and the relationship to deep learning goals. The authors pose questions for developers of SGs in each category, which help to guide the development process, including the selection of an appropriate learning theory.

Lim et al. (2014) attempted to better define the role of narrative in serious games by proposing the concept of narrative serious game mechanics (NSGMs). The authors present three types of NSGM, exposition, guidance and reflection and feedback, and provide details and guidelines on how each can be implemented in an SG.

The concept of serious game mechanics (SGMs) was also utilised by Arnab et al. (2015), who proposed that a serious game is composed of a set of SGMs, each of 
which is formed from a learning mechanic (LM) and a game mechanic (GM). The resulting model LM-GM demonstrates how these mechanics can be selected and paired to achieve a successful balance between learning and entertainment.

Carvalho et al. (2015) presented the activity theory-based model of serious games (ATMSG), proposing that the use of an SG can be described with activity theory, specifically through activities for gaming, learning and instruction. Each activity is composed of lower level actions, tools and goals, which are implemented using different game mechanics, designs and rules. Furthermore, the authors also suggested the model could assist in the design of SGs utilising a service-oriented architecture (Carvalho et al. 2015).

Finally, Tang and Hanneghan (2014) proposed a methodology to apply to the entire design and development process of a serious game. They performed a review of existing educational games and learning theories and incorporated them into traditional game design methodologies to produce a methodology for educational game design, formed from 13 steps organised into 3 phases-plan, prototype and finalise.

\section{A framework for embedding informational content in core game mechanics}

Despite the plethora of different frameworks for designing serious games, there is still relatively little guidance for how to design the low-level mechanics and content of an SG for a given set of learning objectives. Hall et al. (2014) highlighted this gap in the literature and proposed a model for embedding learning outcomes in the game core mechanics (according to the Sicart (2008) definition of core mechanics). This was based on theories of human-computer interaction (HCI) and instructional objective design, using a loop formed from goals, choices, actions, rules and feedback. The authors applied the model to the design of a serious game for teaching competence in occupational health and safety and demonstrated the effectiveness of the resulting game compared with an equivalent e-learning course (Hall et al. 2016).

Despite the effectiveness of this model, it was applied to a skill-based learning objective where the game mechanics could replicate situations requiring application of that skill. For more information-based learning objectives such as heritage, we believe that this on its own is insufficient. For example, if the learning objective of an SGH is to teach players about a specific historical activity, one could imagine two game designs. In the first, the player might answer numerous detailed and indepth questions about the activity, and their total score could be measured. In the second, the player could carry out the historical activity for themselves through the game mechanics and explore the possibility space of the activity in an experimental manner. One might conclude that the second design sounds superior, yet Hall et al.'s (2014) model is unable to offer insight into why. We propose this notion of exploration of historical activity is crucial and will integrate it into a methodology for designing $\mathrm{SGsH}$ in a later section. 


\section{A model of heritage content in video games}

A model of the different forms of heritage informational content, their associated learning outcomes, and the ways they can be manifested in the video game medium was presented by Hanes and Stone (2017). This model is shown in Fig. 1 and, in this part of the paper, each constituent element will be briefly outlined and described.

The layout of the model is based on the conceptual framework of serious games presented by Yusoff et al. (2009), selected for its conceptual simplicity and completeness, and due to the authors' additional efforts to validate the framework experimentally (Yusoff et al. 2010).

\section{Potential learning outcomes}

This element is equivalent to the intended learning outcomes element of Yusoff et al.'s (2009) framework, however with a subtle wording change to represent the

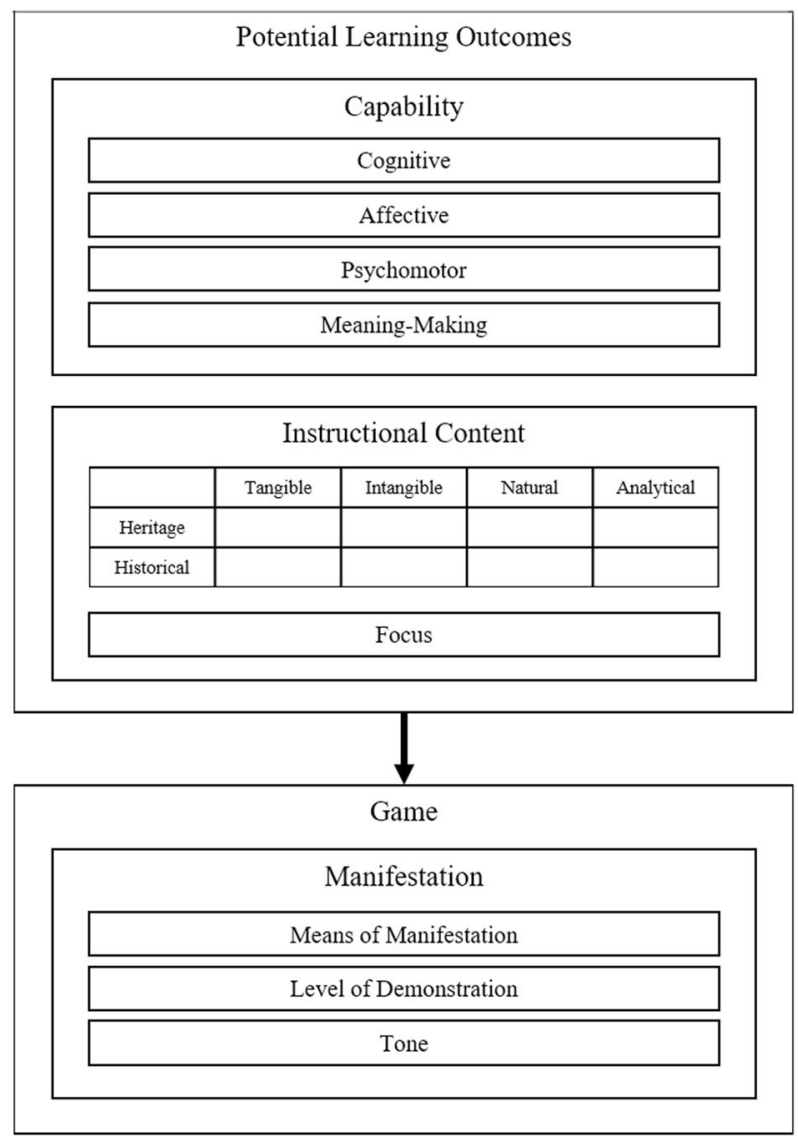

Fig. 1 A model of heritage content in serious and commercial games. (C) 2017 IEEE. Reprinted, with permission, from Hanes and Stone (2017) 
possibility for non-intended learning outcomes, such as those in entertainment video games.

\section{Capability}

This element represents the types of skills and competences gained from playing the game. Yusoff et al. (2009) populated this element with the categories of cognitive, affective and psychomotor capabilities. To these we propose the addition of the paradigm of meaning-making (Silverman 1993, 1995), due to its adoption and success within the museum and heritage domains (e.g. Cohen-Stratyner 2013; Bailey-Ross et al. 2016).

\section{Instructional content}

This element represents the material learned by the player, presented as an eightelement taxonomy according to a heritage-historical dimension and a tangibleintangible-natural-analytical dimension. Heritage information refers to "what existed", whereas historical information refers to "what happened". Therefore, tangible heritage refers to physical artefacts and buildings of cultural value, intangible heritage denotes non-physical articles such as language, ceremonies, customs and beliefs, as in the UNESCO definition (2003). Natural heritage refers to natural landscapes, flora and fauna and analytical heritage represents archaeological and historical processes, research, and analysis conducted within the preceding three heritage categories. Tangible historical refers to historical events, processes and actors whereas intangible historical represents how people reacted to or were affected by those tangible events and processes. Natural historical refers to the historical events and processes associated with natural heritage, and analytical historical denotes the archaeological and historical processes, research and analysis conducted within the preceding three historical categories.

The focus of the instructional content refers to which aspects of the information are included in the manifestation, which are emphasised, which are omitted, and even which are entirely falsified, which may be done for the sake of gameplay when there is a priority on entertainment rather than educational value and historical accuracy. This idea was originally demonstrated by Schaller (2014) for the game Assassin's Creed IV: Black Flag which is set in the Caribbean during the Age of Sail. The game features many historically accurate 3D sword models, which the player can select and fight with. However, the focus of this heritage informational content is not on the manufacture, aesthetics or significance of the artefacts, aspects a museum would typically want to present. Instead, the only information presented for these swords is characteristics such as "speed" and "damage", which are contrived values used for the sword-fighting gameplay, with no true historical analogue. We observe that the focus of the heritage content is the key differentiating factor between heritage content in SGsH and in commercial games, and something educators wishing to use entertainment games in history education should be keenly aware of. Furthermore, focus can have the effect of diluting other content-if it is 
not clearly marked which content has what type of focus, the player may have no way of knowing what content is historically valuable and what is not.

\section{Manifestation}

The informational content must be manifested within the game, although the specific characteristics of this manifestation, what Chapman (2016) refers to as the "ludic aesthetics of historical description", can take many forms. Having reviewed many examples of SGsH and heritage entertainment games, these characteristics have been distilled down to three principal properties- the means of manifestation, level of demonstration and tone, although this is not intended to provide an exhaustive description.

\section{Means of manifestation}

Verbal - the heritage information is manifested through graphical text, aural speech or a combination. This is most commonly found through information boxes within the game's user interface or through the speech of game characters and voice-overs.

Graphical-the heritage information is manifested through visual means, either photographic or through 2D or 3D graphics, and can be either static or animated. This is the most commonly utilised means of manifestation and can be used to immediately convey large quantities of information, as well as create games that are immediately eye-catching and engaging. However, graphical content can also be the most resource-intensive to create, especially for accurate 3D recreations of artefacts or historical sites. Graphical representation is the primary means used by Chapman's (2016) realist simulation style.

Aural - the heritage information is manifested through sound effects and music. This means is most commonly used for game ambience, nevertheless it can play an important role in creating a strong sense of presence for the player (Larsson et al. 2010).

Mechanical-the heritage information is manifested through the game's mechanics and player interactions. Relevant here is the theory of Procedural Rhetoric, most frequently attributed to Bogost (2007), which describes the way in which games are able to transfer information, often in a rhetorical manner, through their systems, rules and goals. Bogost (2005) specifically discusses the potential for the procedural rulesets of video games to present historical systems and processes, with the example of the Civilization game series. However, some authors have argued against the efficacy of the theory in the heritage domain (Champion 2015) and generally (Sicart 2011). Mechanical representation is the primary means used by Chapman's (2016) conceptual simulation style.

\section{Level of demonstration}

The level of demonstration denotes whether the manifestation is presented in a demonstrative and literal way, or an abstracted and metaphorical technique. Chapman (2016) notes that realist simulation style games make considerable use of 
demonstrative presentation to reconstruct history "as it was" to the player, whereas conceptual simulation style games may present information in an abstract manner. The reasons behind this distinction may be due to the focus on the heritage informational content; if macro-scale historical processes are to be focused upon rather than the effects of individual actors, it may make sense from a game design perspective to reduce the quantity of information presented to the player by abstracting the presentation style. Furthermore, abstracted manifestation can be an effective method of presenting violent or otherwise inappropriate subject matter to younger players, an approach utilised by Christopoulos et al. (2011).

\section{Tone}

Tone denotes the level of affect used in the manifestation of the content. Within the field of heritage, the use of player empathy is a common approach, and Kidd (2015) performed a critical evaluation of affective design within museum games, highlighting the importance of cognitive empathy and affective learning.

\section{Examples of the model in serious games for intangible heritage}

For examples of the different elements of the model in SGsH and in commercial historical games, we direct the reader to Hanes and Stone (2017). However, one area focused on less in that publication was the presentation of intangible heritage through serious games. This area is of particular interest because of the desire to preserve rich bodies of intangible heritage and traditions and also because of opportunities to cause attitudinal changes in the player towards other cultures through affective learning and the use of empathy (Huang and Tettegah 2014). Presentation of intangible heritage poses challenges for serious game designers, due to its complex, context-dependent nature with less reliance on the audio-visual channel compared with tangible heritage. Furthermore, advances in the capture, storage and analysis of intangible heritage (e.g. Doulamis et al. 2017; Aristidou et al. 2017) are creating large bodies of rich and complex data which now requires effective means of presentation for serious games.

The SGH i-Treasures (Dagnino et al. 2015) aims to achieve psychomotor learning outcomes for intangible heritage, such as folkloric dance, by manifesting the content as a combination of animated 3D character models (sometimes known as "4D" due to 3D assets with an added temporal dimension) and video footage of professionals performing the dance movements. After observing this presentation, the player must imitate the performance, and through instruments such as Kinect depth sensors, the game is able to rate the quality of the player's performance and give feedback for improvement.

The SGH Discover Babylon (Lucey-Roper 2006) aims to raise awareness of the cultures, beliefs and activities of ancient Mesopotamian civilisations. The game environments are made up of static 3D representations of architecture and tangible heritage, with information points scattered throughout, which present the intangible heritage associated with that part of the environment through a purely textual manifestation, in a manner somewhat similar to a typical museum display. For 
example, next to the 3D ziggurat building in the ancient city of Ur there is an information point describing which members of society were permitted to worship at the ziggurat shrine and how the rest of society would worship the gods.

Finally, ICURA (Froschauer et al. 2010) is an SGH that aims to raise awareness and interest in Japanese etiquette and culture. The authors state their intention to utilise constructivist learning principles by allowing players an active role in the game's learning process, by combining presentation of intangible heritage through text and also through the gameplay mechanics. For example, one of the characters explains through an in-game email that people must wear slippers when entering a Japanese temple. This information is replicated in the mechanics, whereby if the player has not interacted with the slippers to put them on, a guard NPC will refuse them entry to the temple.

\section{Appropriate selection and design of heritage content in serious games}

An important aspect of heritage content in serious games, especially during the design and development process, is the appropriateness of the manifestation, and its various characteristics, for the given learning objectives. An appropriate manifestation is one that sufficiently affords and supports the learning objectives for the target user group within the target context of usage, while also meeting the requirements for gameplay experience, and the limitations of development resources and budget.

To compare the SGH examples given above, i-Treasures (Dagnino et al. 2015) utilises high fidelity 3D character animations combined with video footage, which is necessary for the player to be able to learn and perform the intricate physical movements and emotive expressions of the dance steps. Discover Babylon (LuceyRoper 2006), on the other hand, aims to raise general awareness and interest of ancient Mesopotamian cultures. The game features detailed 3D environments to effectively convey spatial information of Mesopotamian cities, however it was decided, perhaps due to budgetary restrictions, that many of the cultural activities that take place within those environments should be presented through brief textual information. ICURA (Froschauer et al. 2010) aims to raise general awareness and interest in Japanese culture for foreign visitors and most of the informational content can be sufficiently conveyed through brief textual information, especially through the use of an in-game helper agent. However, engagement of the player with the content was also a priority, therefore some of the content was also manifested through the game's mechanics.

\section{Analysing heritage content in commercial games}

As McCall (2016) discussed, for educators wishing to incorporate commercial historical video games in their lessons, it is important to have detailed knowledge of the content and mechanics of the games in question and where they are strong or weak at "simulating the history". This notion is equivalent to the third dimension of De Freitas and Oliver's (2006) 4D Framework, the internal representational world 
of the game. We propose that the model previously published and described earlier in this paper is a feasible paradigm through which to perform such analysis.

In this part of the paper we demonstrate this process for the commercial historical game Valiant Hearts: The Great War (Ubisoft Entertainment SA 2014), a singleplayer puzzle adventure game set in the First World War, told from the perspectives of multiple characters. This game has been selected because of its suitability for younger audiences (allocated a $12+$ PEGI rating), ${ }^{1}$ its positive critical reception with $77 \%$ average critic score based on 48 reviews on the critic aggregator Metacritic (2014), and because it elegantly demonstrates some of the aspects of the heritage content model already discussed.

The game is set across multiple levels, in each of which the player assumes the role of one of the central characters and must control the character to solve puzzles in order to progress in the game and unfold the narrative. The narrative is mostly told through cutscenes between each level, all presented through a cartoon art style (Fig. 2). During the gameplay, the player's character can die, causing an instantaneous restart. However, the player never kills enemies, who must instead be knocked unconscious, "sneaked around", or frightened away. Furthermore, there is also a section of the game menu labelled "historical facts", which is split into two sub-sections- "facts" and "items". Most of the heritage content that the player encounters during gameplay has a corresponding entry in the historical facts section. It is clearly intended that the content in the gameplay sections is somewhat simplified and often not historically accurate. In contrast, the historical facts section is intended to be more historically robust, with textual descriptions closer to what would be found in a museum, and the user interface (UI) bearing the logos of two partnered organisations-Mission Centenaire 14-18, ${ }^{2}$ a WWI centenary programme, and Apocalypse World War 1 (Arnaud 2014), an historical documentary series. This is an interesting approach to overcome the issue of inaccurate content diluting more accurate content, as discussed earlier, although the player is never required to look at the historical facts section of the game. Table 1 shows an enumeration of the heritage content from the first level of the game, performed using the proposed model.

It is proposed that such an analysis as that shown in Table 1 should form the first stage of using any commercial historical video game in a history education context. The informational content that is defined through this methodology, and the characteristics of the specific manifestation within the game, should help to inform the choice of game and choice of approach taken within the learning context according to the desired learning outcomes. Use of such games in history education should then follow the guidelines and best practices outlined by the research community, both for implementation (Squire 2005; McCall 2016) and evaluation (Connolly et al. 2008; Hainey et al. 2010).

\footnotetext{
${ }^{1}$ https://pegi.info/.

${ }^{2} \mathrm{http} / / /$ centenaire.org/en.
} 


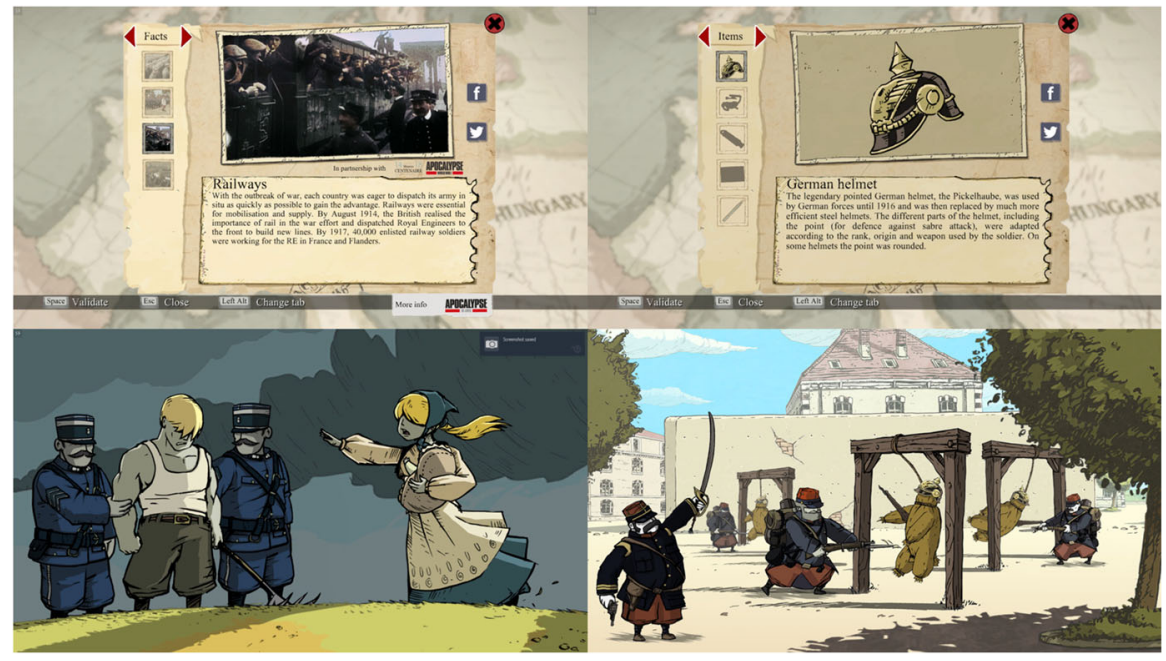

Fig. 2 Screen captures from Valiant Hearts. Top-left clockwise: the "facts" section of the "historical facts" menu; the "items" section of the "historical facts" menu; the gameplay; the cutscenes presenting the game narrative. (C) 2014 Ubisoft, printed with permission

\section{Designing serious games for heritage}

\section{Approach}

As previously noted, it is proposed that for SGsH the state-of-the-art in SG conceptual frameworks is insufficient for providing adequate guidelines for embedding a given set of heritage learning outcomes in a serious game. A novel methodology will therefore be presented that addresses the specific requirements of heritage information.

Having reviewed the literature surrounding the design of SGsH and the use of technology to enhance visitor learning in museums, a model presented by Kaptelinin (2011) was selected as the most appropriate for approaching the embedding of heritage content in a serious game application. This model uses activity theory and the concept of bridging activity contexts (defined as "through observation, imagination, inquiry and/or physical interactions, visitors [opening] up their activity contexts and reach out to meanings, values and personal experiences revealed through understanding [artefact]-related activity contexts") to represent the use of information technology by visitors in a museum setting to view and learn about artefacts (Kaptelinin 2011). For the purposes of this part of the paper, a brief explanation will be given of the relevant concepts of activity theory.

\section{Background on activity theory}

Activity theory originated in Soviet psychology and has now expanded into a wideranging field. For a more detailed account of the history of activity theory and its 


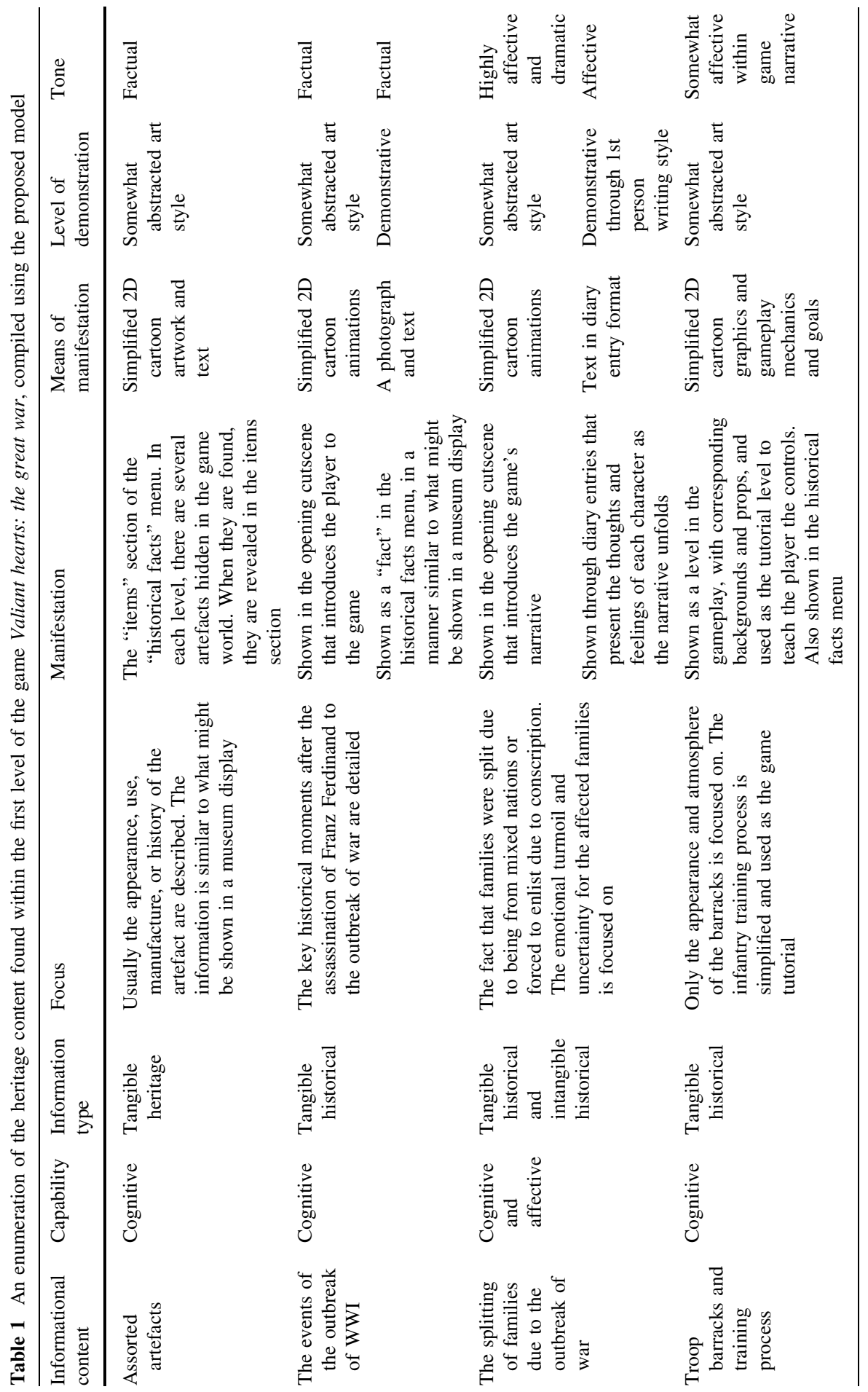




\begin{tabular}{|c|c|c|c|c|}
\hline$\stackrel{\mathscr{0}}{\stackrel{0}{*}}$ & 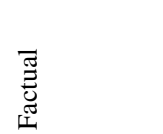 & 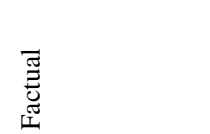 & 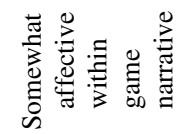 & 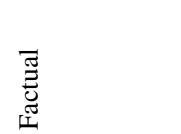 \\
\hline 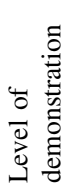 & 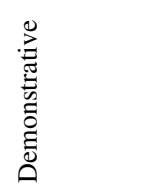 & 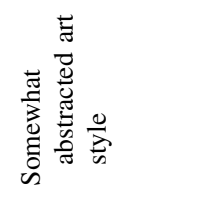 & 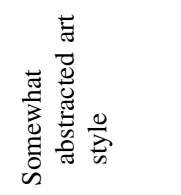 & 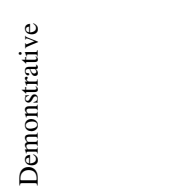 \\
\hline 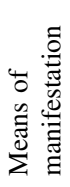 & 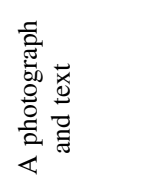 & 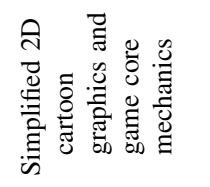 & 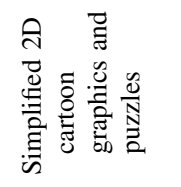 & 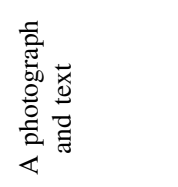 \\
\hline 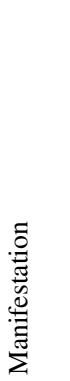 & 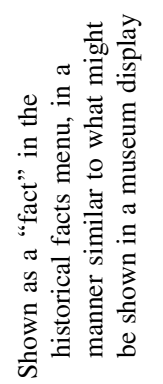 & 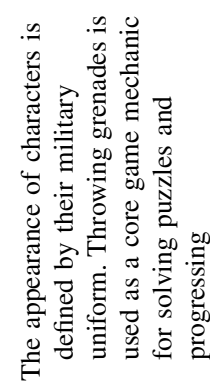 & 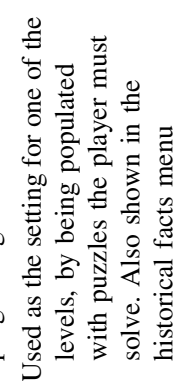 & 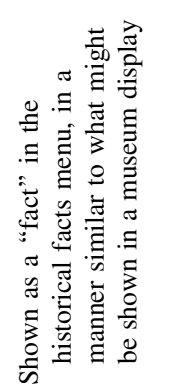 \\
\hline $\begin{array}{l}气 \\
\bar{U} \\
\text { I. }\end{array}$ & 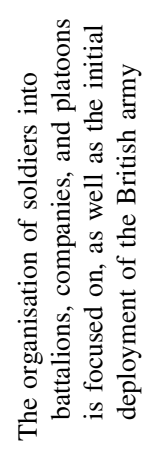 & 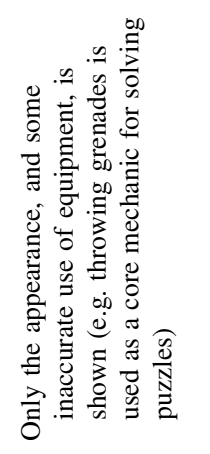 & 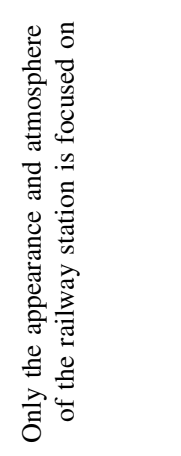 & 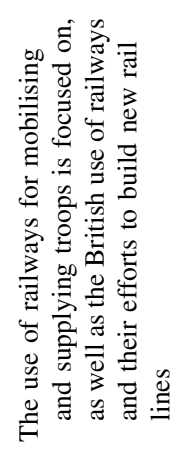 \\
\hline 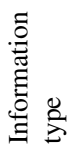 & 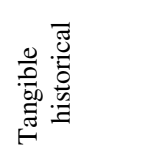 & 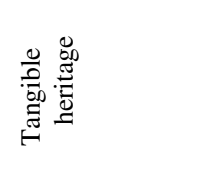 & 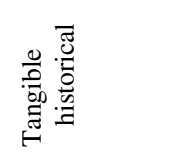 & \\
\hline 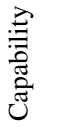 & 泀 & 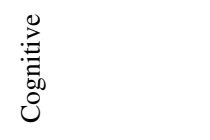 & 泀 & \\
\hline 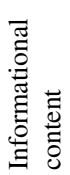 & 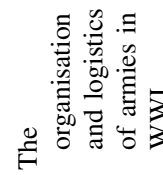 & 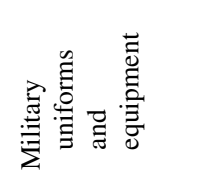 & 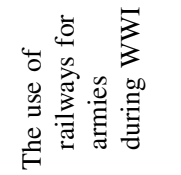 & \\
\hline
\end{tabular}


applications in HCI, the reader is referred to Kaptelinin and Nardi (2006). The most basic element of activity theory is the activity context which consists of a subject who carries out an activity by interacting with an object using mediating tools, which are either external in nature (such as a physical tool) or internal (such as plans and mental models). Leontiev (1978) proposed that each activity is directed by a motive and that an activity is made up of actions, each of which is performed according to a known goal, and each of these actions is made up of operations, each constrained by certain conditions. This system is shown in Fig. 3.

Engeström (1987) extended the concept of activity contexts into activity networks to include a social dimension, shown in Fig. 4. Furthermore, Engeström (1990) also defined the concepts of upward and down contextualisation of activity as the exploration of the high levels (the motives, values, context) and the low levels (actions, operations, goals and conditions) of the activity, respectively.

\section{Activity theory for modelling museum and SGH learning}

Kaptelinin (2011) suggested that in activity theory terminology, a museum visitor (the subject) uses IT (the tool) to view an artefact (the object) to facilitate their meaning-making (the motive). The purpose of the museum activity context is for the artefact to be bridged to the historical activity context, where the historical activity was originally carried out. Typically, the museum artefact might have been used as either a tool in the historical activity context or as an object. Bridging can occur in either case. It is proposed in this paper that a similar conclusion can be drawn regarding serious games for heritage, where a player plays a serious game to observe manifestations of the heritage content, thereby creating a bridge between the heritage manifestation and the historical activity context. Due to the expansive possibilities of what can be manifested through the video game medium, this bridging could arguably occur with any aspect of the historical activity context, and not just the tool or object. These concepts are shown in Fig. 5.

\section{Methodology for designing serious games for heritage}

These concepts grounded in activity theory can therefore be implemented into a novel methodology to give practical guidelines to the designers of an SGH for how to improve the activity context bridging that takes place. This methodology will explore each component of the historical activities within the heritage informational content and how it can be manifested within the serious game.

The methodology is presented in Table 2. The principal aim is to represent the historical activity within the game, and to engage the player with the activity through the game mechanics. The first step of the methodology is to define the historical activities in the heritage content to be presented through the serious game. The next step is, for each historical activity, to define the constituent elements of that activity, as listed in Table 2. The final step is, for each constituent activity element, to explore how that element can be manifested in the serious game using the previously described model of heritage content. This process is assisted by implementation questions shown alongside each activity element. It must be 


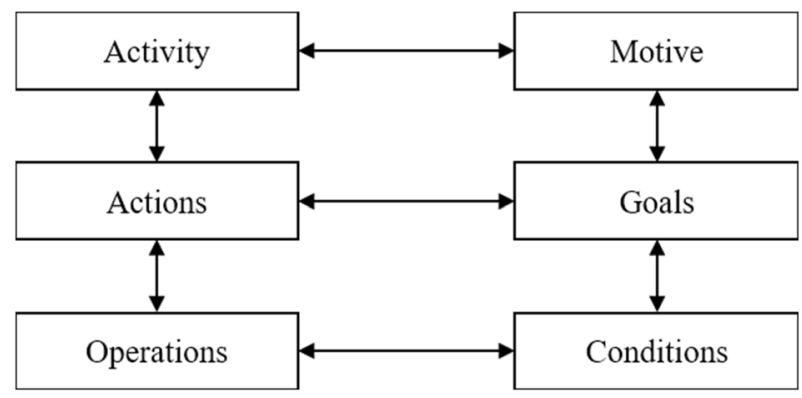

Fig. 3 Activities are directed by a motive and are made up of actions, performed to achieve goals, which are made up of operations performed within conditions. Reprinted from Kaptelinin and Nardi (2006, Fig. 3.4, p. 64). (C) 2006 Massachusetts Institute of Technology, by permission of The MIT Press

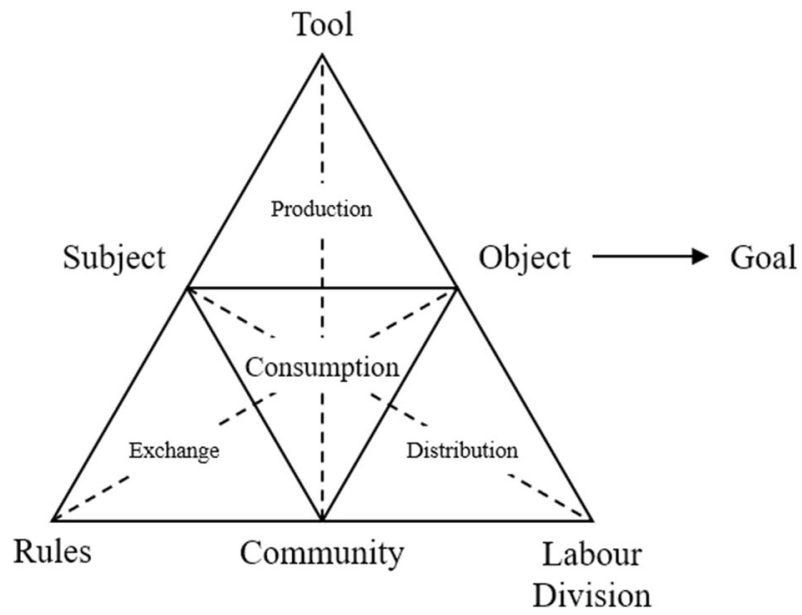

Fig. 4 The activity network extends the concept of activity contexts with the inclusion of the community, which interacts with the subject through social rules and with the object through the division of labour. (C) 1987, 2015 Yrjö Engeström, published by Cambridge University Press. Reprinted, with permission, from Engeström (2014)

stressed that this methodology has been designed to be utilised alongside other conceptual frameworks and game design methodologies in a complimentary manner, since it offers explorative and constructive insight without imposing additional constraints or limitations on the serious game designer.

\section{Methodology integration and validation}

For validating the model of heritage content and the proposed methodology for designing serious games for heritage, it is recommended that the methodology is applied to the design and development of an SGH through integration with an appropriate SG design, implementation and evaluation process. In the following section, we discuss how the methodology could be integrated into the relevant parts 

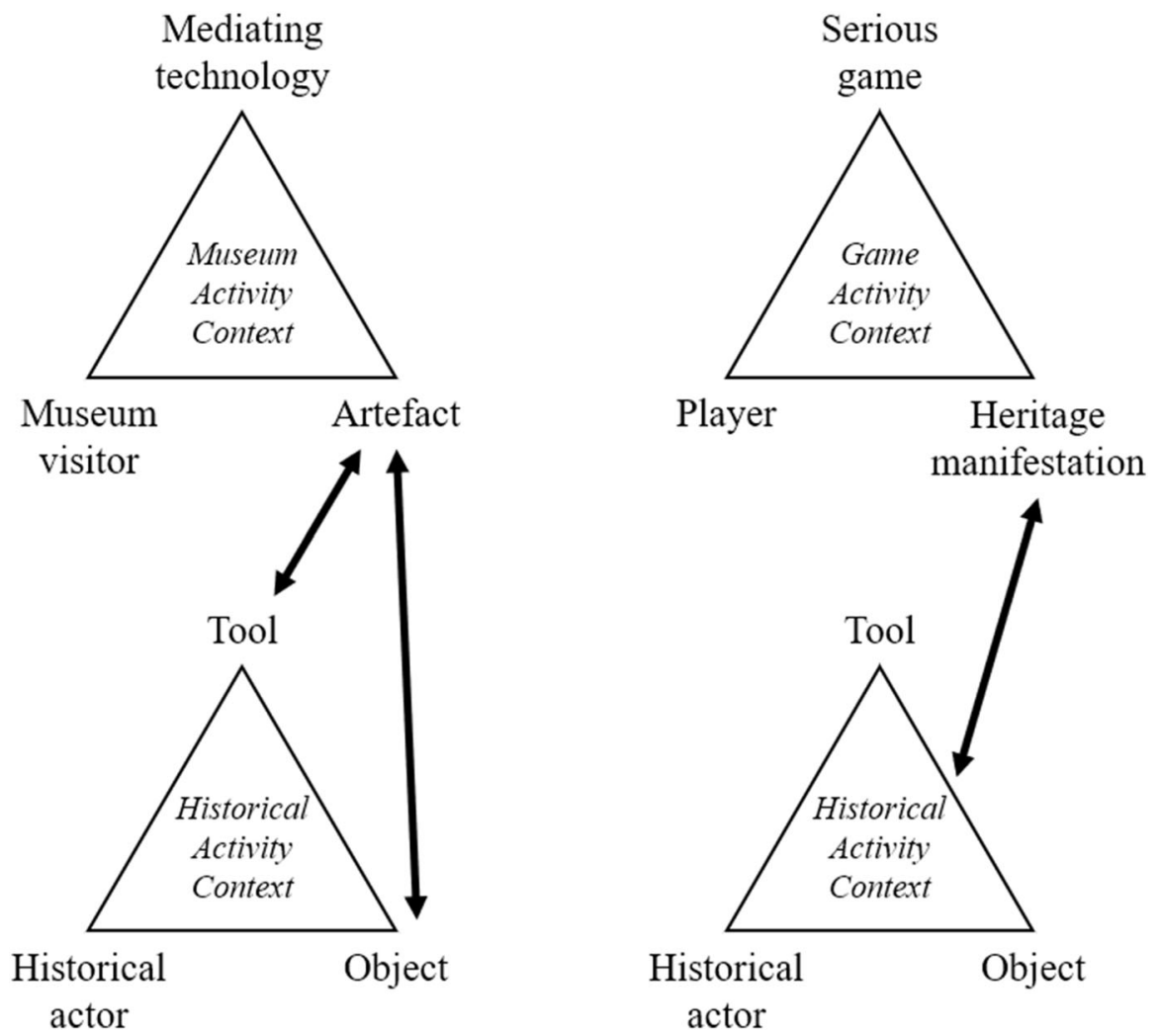

Fig. 5 Left: a museum visitor views an artefact using some mediating technology, thereby bridging with the tool or the object in the historical activity context. (C) 2011 IEEE. Reprinted, with permission, from Kaptelinin (2011). Right: a player plays a serious game to observe manifestations of heritage, thereby bridging with any part of the historical activity context

of the 13 steps of the serious game design process presented by Tang and Hanneghan (2014), incorporating the SGH evaluation processes proposed by Birchall et al. (2012). The framework of Tang and Hanneghan (2014) was chosen for this process because it is one of few SG frameworks that considers the whole serious game development process, built on methods and best practices established and refined by the commercial videogame industry. The framework of Birchall et al. (2012) was chosen because it presents a clear review of the state-of-the-art in evaluation methods for serious games for heritage and when and how to use them.

- Step 3-Identify learning activities for learning objectives Identifying and designing the learning activities can be assisted using the presented methodology to identify and explore the historical activities present within the heritage informational content for the learning objectives and how they can be represented within the serious game. The Activity Theory-Based Model of Serious Games (Carvalho et al. 2015) is identified as an additional approach for defining the gaming and learning activities. 
Table 2 A methodology for bridging between the heritage manifestation and the historical activity context when designing serious games for heritage

\begin{tabular}{lll}
\hline $\begin{array}{l}\text { Activity } \\
\text { Element }\end{array}$ & Activity relationship & Serious game implementation questions \\
\hline $\begin{array}{c}\text { Activity } \\
\text { context }\end{array}$ & $\begin{array}{c}\text { The historical activity in question, } \\
\text { being carried out in a given time } \\
\text { and place }\end{array}$ & $\begin{array}{c}\text { Can the player perform this activity? } \\
\text { Is this activity performed by non-player actors? } \\
\text { How is progress in this activity manifested in } \\
\text { the game? } \\
\text { How is a successful/unsuccessful outcome } \\
\text { manifested in the game? What happens? } \\
\text { How does this activity relate to other historical } \\
\text { activities? }\end{array}$
\end{tabular}

Subject The actor who carries out the activity

Can the player assume the role of the subject?

How can the player interact with the subject?

How is the subject manifested in the game?

Object The focus of the activity

How can the player interact with the object?

How is the object manifested in the game?

Tools The (tangible and intangible) tools used to carry out the activity

How can the player interact with the tools?

How do the tools affect the performance of the activity?

How are the tools manifested in the game?

Motives The motives and values that drive the actors to carry out the activity

How do the motives affect the performance of the activity?

How are the motives manifested in the game?

Actions and The actions and their respective goals goals that make up the activity

How do the actions of the activity relate to the actions undertaken by the player?

How does the completion of the actions affect the performance of the activity?

How are the actions manifested in the game?

How are the goals manifested in the game?

Operations The operations and their respective and conditions that make up each conditions action and goal

How do the operations of the activity relate to the operations undertaken by the player?

How does the completion of the operations affect the performance of the related action?

How are the operations manifested in the game?

How are the conditions manifested in the game?

Community The greater community which the subject carries out the activity within

Rules Social rules that connect the subject to the community

How is the community manifested in the game?

How do the rules affect the performance of the activity?

How are the rules manifested in the game?

Labour Systems that distribute the activity division amongst the community

How do the systems of labour division affect the performance of the activity?

Is labour division controlled by the player?

How is labour division manifested in the game? 
- Step 6-Design game mechanics for learning activities The presented methodology can be used to assist in the design of the core game mechanics by considering the historical activities and how each element of those activities can be manifested in the game mechanics, such that activity context bridging is maximised. It is observed that the methodology presented by Hall et al. (2014) would also be effective at this stage to ensure the learning objectives are successfully embedded in the game mechanics.

- Step 7-Design game components and associated behaviours The presented methodology with the model of heritage content can be used to assist in the design of each game component and how the heritage informational content and each component of the historical activities are manifested in the serious game, and the systems and behaviours associated with them.

- Step 10-Evaluate prototype against learning objective As each level and segment of the game is prototyped, formative evaluation should take place, as detailed by Birchall et al. (2012), whereby the prototypes are tested with members of the target audience using methods such as observations and questionnaires. During this evaluation, according to the proposed methodology, attention should be paid to the extent to which the gameplay and narrative of the serious game create cognitive empathy (Kidd 2015) towards the historical actors and the activities they partake in.

- Step 13-Quality assurance (QA) test on educational game Once the serious game is fully developed it can be play-tested in its entirety. As in the evaluation of the prototypes for each level, methods such as observations, questionnaires and interviews can be used to ascertain the extent to which the holistic game experience can create a sense of cognitive empathy and successfully bridge the heritage manifestations with the historical activity context.

Furthermore, summative evaluation can be carried out post-launch using methods such as in-game analytics, player statistics, questionnaires and interviews (Birchall et al. 2012). This is to further evaluate the effectiveness of the SGH at presenting and exploring historical activity, achieving its learning objectives, and being an engaging game experience, and finally also to inform future SGH projects. Of these methods, in-game analytics has received particular interest in recent years for serious games (also known as gaming learning analytics or GLA) and can offer powerful insights by detecting a player's in-game actions, aggregating the data for many players over many play sessions, and analysing the data (Freire et al. 2016). An interesting avenue for further research work will be to further integrate datadriven analytics into the evaluation of how well an SGH can bridge activity contexts. Other relevant references for the design process include the concept of intrinsic and extrinsic design (Schaller 2014), a framework for matching game genres with learning outcomes (Sherry and Pacheco 2006), and a framework for selecting game engines for serious game projects (Petridis et al. 2012).

The approach described in this section is currently being implemented for a webbased game presenting material on ancient Mesopotamian history. Early results so far have confirmed that the proposed methodology is a useful tool for both analysing and designing a serious game for heritage. The methodology has been particularly 
effective at identifying important aspects of the informational content and streamlining the process of generating game design concepts based on this content. The presented model of heritage content has also been useful for exploring how the identified elements of the historical activities can be manifested within the game, especially when the design project is more restricted by the development resources and budget.

\section{Strengths and weaknesses of the proposed methodology}

We believe the greatest strength of the proposed methodology is through its use of activity theory. Activity theory has already been shown to be an effective paradigm for modelling gameplay, learning and instruction within serious games (Carvalho et al. 2015) and the methodology extends this "common language" to the heritage field. This should help to facilitate communication between experts in the heritage, game design and pedagogy domains. Another strength of the methodology is that it helps to generate and explore possible serious game designs and is particularly useful at the concept generation phase of development. The final strength is that it encourages heritage content to be situated naturally within the serious game, by embedding more elements of the historical activity within the game in a meaningful and unified manner, rather than being isolated, leading to a more seamless blend of gaming and learning.

The primary weakness of the methodology is that it encourages creators of SGsH to create serious game designs based off of the heritage content. While this approach, what Schaller (2014) describes as intrinsic SGH design, has its advantages, it also entails more risk and is more resource-intensive. Finally, the methodology is not prescriptive and cannot automate the process of generating serious game designs from given heritage learning content; it still requires the challenging work and imaginative input of game designers, heritage experts and pedagogical specialists working together.

\section{Conclusions}

In this paper, an overview of the current state-of-the-art in definitions and conceptual frameworks for both serious games for heritage and commercial historical games has been presented. The overview has highlighted a significant lack of definitions for these games, in particular from the perspective of examining individual game mechanics and content, as opposed to examining the game as a single component. Details have also been provided of a model of heritage content to describe these games, originally presented by Hanes and Stone (2017).

It has been shown how this model can be used to enumerate and explore the heritage content contained within a commercial historical game designed for entertainment with no pre-defined learning objectives, making it exploitable in the field of historical education. History educators wishing to use video games in their course material now have an additional analysis tool to assist in the selection of an appropriate historical game, and to help design the instructional material around the game content. 
A novel methodology has also been presented to apply the model of heritage content to the design of serious games for heritage, through an approach based on activity theory, maximising the bridging of the manifestations of heritage content in the game with the historical activities the game is attempting to represent. This methodology can be applied alongside other methodologies and frameworks, and it has been shown how it can be integrated into existing serious game frameworks. Designers of SGsH now have an additional tool to assist them in converting heritage informational content and learning objectives into real and feasible serious game designs. Future work in this field will continue to apply the proposed methodology to real-world case-studies of serious games for heritage, and to further investigate how serious game evaluation methodologies, such as gaming learning analytics, can be used to evaluate the extent to which SGsH achieve activity context bridging.

Open Access This article is distributed under the terms of the Creative Commons Attribution 4.0 International License (http://creativecommons.org/licenses/by/4.0/), which permits unrestricted use, distribution, and reproduction in any medium, provided you give appropriate credit to the original author(s) and the source, provide a link to the Creative Commons license, and indicate if changes were made.

\section{References}

Amory, A. (2007). Game object model version II: A theoretical framework for educational game development. Educational Technology Research and Development, 55(1), 51-77.

Anderson, E. F., et al. (2010). Developing serious games for cultural heritage: A state-of-the-art Review. Virtual Reality, 14(4), 255-275.

Antoniou, A., et al. (2013). An approach for serious game development for cultural heritage: Case study for an archaeological site and museum. Journal on Computing and Cultural Heritage (JOCCH), $6(4), 17.1-17.19$.

Aristidou, A. et al. (2017). Emotion control of unstructured dance movements. In Proceedings-SCA 2017: ACM SIGGRAPH/Eurographics Symposium on computer animation. Art. No. a9.

Arnab, S., et al. (2015). Mapping learning and game mechanics for serious games analysis. British Journal of Educational Technology, 46(2), 391-411.

Arnaud, F. (2014). Apocalypse: World war 1.

Bailey-Ross, C., et al. (2016). Engaging the museum space: Mobilizing visitor engagement with digital content creation. Digital Scholarship in the Humanities, 32(4), 689-708.

Beale, I. L., et al. (2007). Improvement in cancer-related knowledge following use of a psychoeducational video game for adolescents and young adults with cancer. Journal of Adolescent Health, 41(3), 263-270.

Bellotti, F., et al. (2012). A serious game model for cultural heritage. Journal on Computing and Cultural Heritage (JOCCH), 5(4), 17.1-17.27.

Birchall, D. et al. (2012). Levelling up: Towards best practice in evaluating museum games. In Museums and the web conference (pp. 11-14). http://www.museumsandtheweb.com/mw2012/papers/ levelling_up_towards_best_practice_in_evaluati.html.

Birchall, D., \& Henson, M. (2011). High tea evaluation report. Wellcome Trust, August.

Bloom, B. S. (1956). Taxonomy of educational objectives, handbook I: The cognitive domain. New York: David McKay Co., Inc.

Bogost, I. (2005). Procedural literacy: Problem solving with programming, systems, and play. Journal of Media Literacy, 52(1-2), 32-36.

Bogost, I. (2007). Persuasive games: The expressive power of videogames. Cambridge: MIT Press.

Boyle, E. A., et al. (2016). An update to the systematic literature review of empirical evidence of the impacts and outcomes of computer games and serious games. Computers and Education, 94, $178-192$. 
Carvalho, M. B., Bellotti, F., Berta, R., et al. (2015a). An activity theory-based model for serious games analysis and conceptual design. Computers and Education, 87, 166-181.

Carvalho, M. B., Bellotti, F., Hu, J., et al. (2015). Towards a service-oriented architecture framework for educational serious games. In 2015 IEEE 15th International conference on advanced learning technologies (ICALT) (pp. 147-151). IEEE.

Catalano, C. E., Luccini, A. M. \& Mortara, M. (2014). Best practices for an effective design and evaluation of serious games. International Journal of Serious Games. https://doi.org/10.17083/ijsg. v1i1.8.

Champion, E. (2015). Game-based learning and the digital humanities. Critical gaming: Interactive history and virtual heritage (pp. 25-60). London: Routledge.

Chapman, A. (2016). Simulation styles and epistemologies. In Digital games as history: How videogames represent the past and offer access to historical practice (pp. 59-89). Routledge.

Chen, S., et al. (2013). A case study of user immersion-based systematic design for serious heritage games. Multimedia Tools and Applications, 62(3), 633-658.

Cheng, M.-T., et al. (2015). The use of serious games in science education: A review of selected empirical research from 2002 to 2013. Journal of Computers in Education, 2(3), 353-375.

Christopoulos, D. et al. (2011). Using virtual environments to tell the story: "The Battle of Thermopylae." In 2011 Third International conference on games and virtual worlds for serious applications (VS-GAMES) (pp. 84-91). IEEE.

Cohen-Stratyner, B. (2013). Authority and meaning-making in the digital era. In Exhibitionist, (Spring) (pp. 22-26).

Connolly, T. M., Stansfield, M. H., \& Hainey, T. (2008). Development of a general framework for evaluating games-based learning. In Proceedings of the 2nd European conference on games-based learning (pp. 105-114). Barcelona.

Connolly, T. M., et al. (2012). A systematic literature review of empirical evidence on computer games and serious games. Computers and Education, 59(2), 661-686.

Copplestone, T. J. (2017). But that's not accurate: the differing perceptions of accuracy in culturalheritage videogames between creators, consumers and critics. Rethinking History, 21(3), 415-438.

Dagnino, F.M. et al. (2015). Serious games to support learning of rare "Intangible" cultural expressions. In 9th International technology, education and development conference (INTED 2015) (pp. 7184-7194). http://www.itd.cnr.it/download/Paper_INTED2015_final-upload_sito.pdf.

Damani, B., Sardeshpande, V., \& Gaitonde, U. (2015). Use of serious games for creating awareness about social enterprises. Journal of Computers in Education, 2(4), 493-511.

De Freitas, S., \& Oliver, M. (2006). How can exploratory learning with games and simulations within the curriculum be most effectively evaluated? Computers and Education, 46(3), 249-264.

Degens, N., Bril, I. \& Braad, E. (2015). A three-dimensional model for educational game analysis and design. In Foundations of digital games.

Dondlinger, M. J. (2007). Educational video game design: A review of the literature. Journal of Applied Educational Technology, 4(1), 21-31.

dos Santos, A. D., \& Fraternali, P. (2015). A comparison of methodological frameworks for digital learning game design. International conference on games and learning alliance (pp. 111-120). Berlin: Springer.

Doulamis, A. et al. (2017). Transforming intangible folkloric performing arts into tangible choreographic digital objects: The terpsichore approach. In International conference on computer vision, theory and applications, VISAPP. Porto, Portugal.

Doulamis, A., et al. (2012). Serious games for cultural applications. In D. Plemenos \& G. Miaoulis (Eds.), Intelligent computer graphics 2011 (pp. 97-115). Berlin, Heidelberg: Springer.

Engeström, Y. (1987). Learning by expanding: An activity-theoretical approach to developmental research. Helsinki: Orienta-Konsultit.

Engeström, Y. (1990). Learning, working, and imaging: Twelve studies in activity theory. Helsinki: Orienta-Konsultit.

Engeström, Y. (2014). Learning by expanding: An activity-theoretical approach to developmental research (2nd ed.). Cambridge: Cambridge University Press.

Freire, M. et al. (2016). Game learning analytics: Learning analytics for serious games. In Learning, design, and technology (pp. 1-29). Cham: Springer. https://doi.org/10.1007/978-3-319-17727-4.

Froschauer, J. et al. (2010). Design and evaluation of a serious game for immersive cultural training. In 2010 16th International conference on virtual systems and multimedia (VSMM) (pp. 253-260). IEEE. 
Gagne, R. (1985). The Conditions of Learning (4th ed.). New York: Holt, Rinehart \& Winston.

Girard, C., Ecalle, J., \& Magnan, A. (2013). Serious games as new educational tools: How effective are they? A meta-analysis of recent studies. Journal of Computer Assisted Learning, 29(3), 207-219.

Gunter, G., Kenny, R., \& Vick, E. (2006). A case for a formal design paradigm for serious games. The Journal of the International Digital Media and Arts Association, 3(1), 93-105.

Gunter, G. A., Kenny, R. F., \& Vick, E. H. (2008). Taking educational games seriously: Using the RETAIN model to design endogenous fantasy into standalone educational games. Educational Technology Research and Development, 56(5-6), 511-537.

Hainey, T., Connolly, T. \& Boyle, L. (2010). A refined evaluation framework for games-based learning. In Proceedings of the 4th European conference on games based learning. Copenhagen, pp. 97-105.

Hall, J. V., Wyeth, P. A. \& Johnson, D. (2014). Instructional objectives to core-gameplay: A serious game design technique. In Proceedings of the first ACM SIGCHI annual symposium on computer-human interaction in play (pp. 121-130). ACM.

Hall, J., Wyeth, P. \& Johnson, D. (2016). Creating authentic experiences within a serious game context: Evaluation of engagement and learning. In Joint International Conference on serious games (pp. 55-66). Springer.

Hanes, L. \& Stone, R. (2017). A model of heritage content in serious and commercial games. In 20179 th International Conference on virtual worlds and games for serious applications (VS-Games) (pp. 137-140). Athens: IEEE.

Huang, W.-H. D., \& Tettegah, S. (2014). Cognitive load and empathy in serious games: A conceptual framework. In J. Bishop (Ed.), Gamification for human factors integration: Social, education, and psychological issues (pp. 17-30). IGI Global: Hershey.

Ibáñez, B. C., Marne, B. \& Labat, J.-M. (2011). Conceptual and technical frameworks for serious games. In Proceedings of the 5th European conference on games based learning (pp. 81-87).

Jacobson, J., Handron, K., \& Holden, L. (2009). Narrative and content combine in a learning game for virtual heritage. Distance Education, 9(2), 7-26.

Kaptelinin, V. (2011). Designing technological support for meaning making in museum learning: An activity-theoretical framework. In 2011 44th Hawaii International conference on system sciences (HICSS) (pp. 1-10). IEEE.

Kaptelinin, V., \& Nardi, B. A. (2006). Acting with technology: Activity theory and interaction design. Cambridge: MIT Press.

Keller, J. M. (1983). Motivational design of instruction. In C. M. Reigeluth (Ed.), Instructional design theories and models: An overview of their current status (pp. 383-434). New York: Lawrence Erlbaum.

Kelly, L. \& Bowan, A. (2014). Gamifying the museum: Educational games for learning. In Museums and the web Asia. http://mwa2014.museumsandtheweb.com/paper/gamifying-the-museum-educationalgames-for-learning/.

Kidd, J. (2015). Gaming for affect: Museum online games and the embrace of empathy. Journal of curatorial studies, 4(3), 414-432.

Kim, S., et al. (2017). Educational games and students' game engagement in elementary school classrooms. Journal of Computers in Education, 4(4), 395-418.

Krathwohl, D. R., Bloom, B. S., \& Bertram, B. M. (1973). Taxonomy of educational objectives, the classification of educational goals. Handbook II: Affective domain. New York: David McKay Co., Inc.

Landers, R. N. (2014). Developing a theory of gamified learning: Linking serious games and gamification of learning. Simulation and Gaming, 45(6), 752-768.

Larsson, P., et al. (2010). Auditory-induced presence in mixed reality environments and related technology. The engineering of mixed reality systems (pp. 143-163). London: Springer.

Leontiev, A. N. (1978). Activity, consciousness and personality. Englewood Cliffs: Prentice-Hall.

Lepe-Salazar, F. (2015). A model to analyze and design educational games with pedagogical foundations. In Proceedings of the 12th International Conference on advances in computer entertainment technology. New York: ACM.

Lim, T. et al. (2014). Narrative serious game mechanics (NSGM)-Insights into the narrativepedagogical mechanism. In International conference on serious games (pp. 23-34).

Lucey-Roper, M. (2006). Discover Babylon: Creating a vivid user experience by exploiting features of video games and uniting museum and library collections. In J. Trant \& D. Bearman (Eds.), Museums and the web 2006: Proceedings. Toronto. http://www.archimuse.com/mw2006/papers/lucey-roper/ lucey-roper.html. 
Marne, B., et al. (2012). The six facets of serious game design: A methodology enhanced by our design pattern library. European conference on technology enhanced learning (pp. 208-221). Berlin: Springer.

McCall, J. (2016). Teaching history with digital historical games: An introduction to the field and best practices. Simulation and Gaming, 47(4), 517-542.

Metacritic (2014). Valiant hearts: The great war aggregated reviews. Retrieved March 28, 2018, http:// www.metacritic.com/game/playstation-4/valiant-hearts-the-great-war.

Mortara, M., et al. (2014). Learning cultural heritage by serious games. Journal of Cultural Heritage, 15(3), 318-325.

Pagnotti, J., \& Russell, W. B. (2012). Using civilization IV to Engage students in world history content. The Social Studies, 103(1), 39-48.

Paliokas, I. \& Sylaiou, S. (2016). The use of serious games in museum visits and exhibitions: A systematic mapping study. In 2016 8th International conference on games and virtual worlds for serious applications (VS-Games). IEEE.

Perez-Valle, A., Aguirrezabal, P. \& Sillaurren, S. (2014). Playhist: Play and learn history. Learning with a historical game vs an interactive film. In Euro-Mediterranean Conference (pp. 546-554). Springer.

Petridis, P. et al. (2012). Game engines selection framework for high-fidelity serious applications. International Journal of Interactive Worlds, 2012, 418638.

Schaller, D. T. (2014). Game mechanics and the museum: Designing simple gameplay around complex content. In 18th annual museums and the web conference. Baltimore. http://mw2014. museumsandtheweb.com/paper/game-mechanics-and-the-museum-designing-simple-gameplayaround-complex-content/.

Sherry, J. \& Pacheco, A. (2006). Matching computer game genres to educational outcomes. Electronic Journal of Communication/La Revue Electronique de Communication, 16(1-2). http://www.cios. org/EJCPUBLIC/016/1/01615.HTML.

Shih, J.-L., Jheng, S.-C., \& Tseng, J.-J. (2015). A simulated learning environment of history games for enhancing players' cultural awareness. Interactive Learning Environments, 23(2), 191-211.

Sicart, M. (2008). Defining game mechanics. The International Journal of Computer Game Research, 8(2). http://gamestudies.org/0802/articles/sicart?viewType=Print\&viewClass=Print.

Sicart, M. (2011). Against procedurality. The International Journal of Computer Game Research, 11(3). http://gamestudies.org/1103/articles/sicart_ap.

Silverman, L. (1993). Making meaning together: Lessons from the field of American history. Journal of Museum Education, 18(3), 7-11.

Silverman, L. (1995). Visitor meaning-making in museums for a new age. Curator: The Museum Journal, $38(3), 161-170$.

Squire, K. (2005). Changing the game: What happens when video games enter the classroom? Innovate: Journal of Online Education, 1(6), p.Article 5. http://nsuworks.nova.edu/innovate/vol1/iss6/5.

Tang, S., \& Hanneghan, M. (2014). Designing educational games: A pedagogical approach. In J. Bishop (Ed.), Gamification for human factors integration: Social, education, and psychological issues (pp. 181-198). IGI Global: New York.

Ubisoft Entertainment SA (2014). Valiant hearts: The great war/Soldats inconnus: Mémoires de la Grande Guerre.

UNESCO. (2003). Text of the convention for the safeguarding of intangible cultural heritage. In UNESCO World Heritage (Ed.), The General Conference of the United Nations Educational, Scientific and Cultural Organization (UNESCO). Paris: UNESCO.

Uricchio, W. (2005). Simulation, history, and computer games. In J. Raessens \& J. Goldstein (Eds.), Handbook of computer game studies (pp. 327-338). Cambridge: The MIT Press.

Watson, W. R., Mong, C. J., \& Harris, C. A. (2011). A case study of the in-class use of a video game for teaching high school history. Computers and Education, 56(2), 466-474. https://doi.org/10.1016/j. compedu.2010.09.007.

Yusoff, A. et al. (2009). A conceptual framework for serious games. In ICALT 2009 Ninth IEEE International Conference on advanced learning technologies (pp. 21-23). IEEE.

Yusoff, A., Crowder, R. \& Gilbert, L. (2010). Validation of serious games attributes using the technology acceptance model. In 2010 Second International conference on games and virtual worlds for serious applications (VS-GAMES) (pp. 45-51). IEEE. 
Laurence Hanes is a postgraduate researcher and doctoral candidate at the University of Birmingham and is currently completing his thesis in the design of serious games for presenting ancient Near-Eastern history.

Robert Stone is professor in the Department of Electronic, Electrical and Systems Engineering at the University of Birmingham and is director of the Human Interface Technologies Team. 